HEALTH PSYCHOLOGY REPORT · VOLUME 8(4), 2020 ORIGINAL ARTICLE
Vahid Delshad (1D)

$1 \cdot \mathrm{B}, \mathrm{C}, \mathrm{D}, \mathrm{E}, \mathrm{F}$

Hamidreza Khankeh (D)

$1,2 \cdot \mathrm{A}, \mathrm{E}$

Abbas Ebadi $\mathbb{D}$

$3,4 \cdot B, C, E$

Mariola Bidzan (D)

$5 \cdot \mathrm{A}, \mathrm{F}, \mathrm{E}$
Gholamreza

Ghaedamini

Harouni (D)

$6 \cdot \mathrm{C}, \mathrm{D}$

Marcus Stueck (D)

$7 \cdot \mathrm{A}, \mathrm{F}$

\title{
Psychobiological risk assessment in emergency medical service drivers: study protocol for structural equation modeling
}

\begin{abstract}
BACKGROUND
Job stress can lead to several complications including physical, psychological, and behavioral consequences. Emergency medical services (EMS) drivers are more likely to be exposed to stress than other occupations due to the nature of their job. High-risk driving and stress-related psychological responses can have irretrievable consequences. Therefore, increasing the amount of attention to traffic psychology and periodic psychobiological risk assessment for this group are essential. Hence, this paper tries to present how to develop a validated battery for assessing psychobiological risk assessment (PBRA) based on bio-centric health management (BHM).
\end{abstract}

PARTICIPANTS AND PROCEDURE

The study will be conducted in two methodological and modeling phases: In the methodological phase, the psychometric properties of the Health Cube Battery (HCB) will be analyzed, and in the second phase, researchers will model BHM in Iran using a structural equation model (SEM).

\section{RESULTS}

The study population consists of EMS drivers. The tool that will be developed in this study is an $\mathrm{HCB}$, which consists of different dimensions of BHM.

\section{CONCLUSIONS}

At the end of the study, a standard HCB will be developed and validated. Also, the BHM model will be mapped based on psychobiological risk assessment for EMS drivers. Other researchers can also cross-culturally adapt and use the battery, and the model could be adopted for other contexts and cultures.

KEY WORDS

emergency; drivers; health; stress management; biocentric

ORGANIZATION - 1: Health in Emergency and Disaster Research Center, University of Social Welfare and Rehabilitation Sciences, Tehran, Iran · 2: Department of Clinical Science and Education, Karolinska Institute, Stockholm, Sweden .

3: Behavioral Sciences Research Center, Life Style Institute, Baqiyatallah University of Medical Sciences, Tehran, Iran .

4: Nursing Faculty, Baqiyatallah University of Medical Sciences, Tehran, Iran · 5: Institute of Psychology, University

of Gdansk, Gdansk, Poland · 6: Social Welfare Management Research Center, University of Social Welfare and

Rehabilitation Sciences, Tehran, Iran · 7: DPFA Academy, Group Academy of Work and Health, Leipzig, Germany

aUthors' Contributions - A: Study design - B: Data collection - C: Statistical analysis - D: Data interpretation .

E: Manuscript preparation · F: Literature search · G: Funds collection

CORRESPONDING AUTHOR - Prof. Hamidreza Khankeh, Health in Emergency and Disaster Research Center,

University of Social Welfare and Rehabilitation Sciences, Kodakyar Ave., Daneshjo Blvd., Tehran, Iran,

e-mail: hamid.khankeh@ki.se

to Cite this ARticle - Delshad, V., Khankeh, H., Ebadi, A., Bidzan, M., Ghaedamini Harouni, G., \& Stueck, M. (2020).

Psychobiological risk assessment in emergency medical service drivers: study protocol for structural equation

modeling. Health Psychology Report, 8(4), 453-461. https://doi.org/10.5114/hpr.2020.99455

RECEIVED 26.07.2020 • REVIEWED 21.08.2020 • ACCEPTED 26.08.2020 • PUBLISHED 01.10.2020 


\section{BACKGROUND}

Over the last few decades, the subject of stress has become increasingly popular in the behavioral and health sciences (Hennessy, Jakubowski, \& Leo, 2016). Exposure to major and minor stressful events and chronic health adversities are the primary sources of risk for mental and physical health problems. The psychological processes involved in stress and responses to stress can provide important clues in distinguishing resilient from vulnerable individuals (Deb, Chakraborty, Chatterjee, \& Srivastava, 2008). When exposed to stress, complex cognitive, behavioral, emotional, and biological processes are activated. These processes work together in concert, leading to adaptation through either avoiding or withdrawing from the source of stress (Compas, 2006). Accurate and timely identification of stressors and preparedness to prevent and cope with them are among the fundamental tasks of dynamic individuals (Moradi, Jafari, \& Hendiani, 2016). Occupational stress is highly experienced among ambulance drivers due to the nature of the job and environmental factors such as contextual and cultural aspects (Hajiamini, Cheraghalipour, Azad Marzabadi, Ebadi, \& Norouzi Koushali, 2011).

Prehospital emergency medical services (EMS) plays a vital role in the network of the health system by transferring the patient to the nearest health care center. The prehospital EMS aims at provision of proper treatment in the appropriate place and time by deploying the available resources. The services start with the basic treatment and medical care and end in the emergency department of the hospital (Delshad et al., 2016), which needs resilient staff to manage these stressful situations.

There is an assumption that there is an inherent stress in the EMS drivers, who form an important humanistic component of the ambulance services, and have to face potentially hazardous situations that can result in physical or mental trauma or even death in the course of duty services (Sterud, Ekeberg, \& Hem, 2006). EMS drivers often work for long hours under stressful conditions, witnessing the agonies, physical destruction, and psychological devastation of the vic-

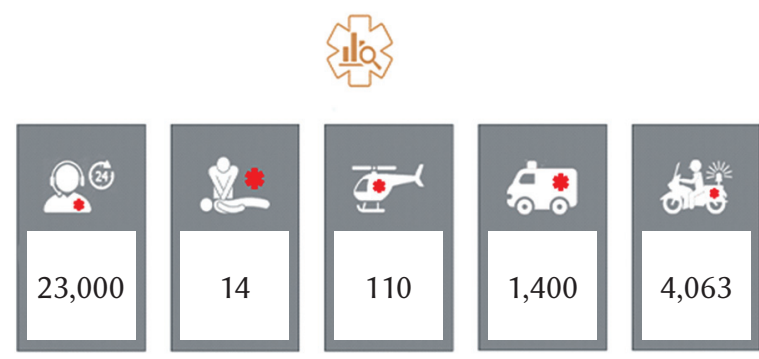

Figure 1. Number of EMS missions in Iran (last 24 hours). tims that may accompany disasters and is also stressful (Koslowsky, Kluger, \& Reich, 2013). Moreover, inappropriate personality traits and coping methods of the drivers can further aggravate their stress in such situations (Rutkow, Gable, \& Links, 2011).

Making wrong decisions or not taking right decisions could turn out to be costly mistakes for the individuals and concerned organizations, especially for EMS drivers.

The EMS workplace has high emotional fluctuations and imposes high pressure (Heydari, Rad, Ghasemi, Sabzevari, \& Rad, 2014).

Iran is a country with a high rate of road traffic incidents' (RTI) fatality and injury and RTI are the second cause of death and disability (Delshad et al., 2018).

As Figure 1 shows, at the time of writing this paper the number of missions in the last 24 hours was 6000 based on the national website report which has been shown (National EMS, 2020). More than half of these missions were in Tehran EMS as the biggest and capital city in Iran with more than 1200 staff members. Every day more than 1000 missions out of 7500 to 9500 calls are usually done. Every year the number of missions and their complexity increase, which imposes a lot of stress for EMS technicians (Entekhab, 2020). Also about $80-85 \%$ of all EMS missions are performed for providing prehospital care and transporting them to medical facilities (Ebrahimian, Seyedin, Jamshidi-Orak, \& Masoumi, 2014).

As has been explained, the emergency medical profession is inherently stressful. Medical emergency technicians experience a lot of stress due to the workplace and conditions of patients. The rate of exposure to stressors affects the staff's health and patients' care.

The results of the Dadashzadeh and Rahmani (2017) study showed that among 45 people who had experienced occupational stressors there was a significant relationship between the rate of exposure with occupational stressors and the number of missions, educational qualifications and employment type. The rate of exposure to stressors was moderately high among emergency medical staff. Identifying the rate of exposure to stressors can be effective in planning and prevention to reduce the amount of burnout and other mental complications among emergency medical technicians (Dadashzadeh \& Rahmani, 2017).

Experiencing a stressful situation and its related complications can decrease the quality of work and reduce the desire to continue serving (Saberinia, Jannat, \& Aminizadeh, 2019).

Since these groups of people are exposed to a high level of stress, this indicated the need for the implementation of comprehensive stress management programs for them (Golmohamadi, Damyar, Mohamadfam, \& Faradmal, 2013).

There is no consensus in previous studies on considering the sources (individual vs. environmental) of job-related stress among EMS drivers and how these 
sources interact. Also, the existing medical guidelines alone have been reported to be insufficient in predicting paramedic drivers' fitness in many aspects (Fox, Bashford, \& Caust, 1992).

Hence, it is essential to clarify the process of stress perception and biopsychological risk assessment in drivers of a specific context to be able to find out about the real pattern of job-related stress that exists among this working group. Therefore, developing a model to achieve this goal is necessary.

The Health Cube is a comprehensive tool for psychobiological risk assessment based on the bio-centric health management (BHM) model, both were developed by Stueck (Stueck, Witruk, \& Utami, 2019b). BHM is a new approach in occupational psychology, which focuses not only on stress-related working conditions and psychological reactions but also on the psychobiological reactions and life-oriented resources (e.g. self-care, health commitment, and affective abilities to support relations). The bio-centric approach is mainly based on the work of Rolando Toro, and was scientifically worked out for health psychology diagnosis and related evidence-based interventions by Stueck (Stueck \& Villegas, 2017; Stueck, Witruk, \& Utami, 2020).

This tool is a flexible scientific and interventional tool for risk assessment, and it ultimately aims to increase the "Health Commitment" and "auto regulative driving competence" among professional drivers (Stueck et al., 2019a). This tool has also been modified for other target groups, such as emergency and disaster management, health management in hospitals, and other professions. The aim of the Health Cube is to identify psychological and physiological risk pat- terns of behavior. The practice of emotion regulation and biofeedback for worker was developed from the Health Cube (Reschke, Kranich, Stoeber, \& Neubauer, 2019; Stueck, 2011).

As Figure 2 shows, the model has 6 levels, 5 of which can be assessed using paper and pencil tools as explained in the first step below.

\section{FIRST STEP: TOOLS VER}

\section{Level 1. External load working conditions}

Disabilities are identified in everyday working life regardless of the type of institution, which can negatively affect the health and wellbeing of employees (Stueck et al., 2019b).

\section{Level 2. Psychobiological reactions}

This level will be explained in the second step separately (because the measurement method is different and it is not assessed using paper and pencil tools) (Stueck et al., 2019b; Schaarschmidt, 2006).

\section{Level 3. Stress consequences}

Repeated onset of overloads (Level 1) and psychobiological stress states (Level 2) result in longer-term adverse effects:

- over-demand reactions, questions on hypersensitivity as signs of incipient fatigue, work-related behavior and experience patterns,

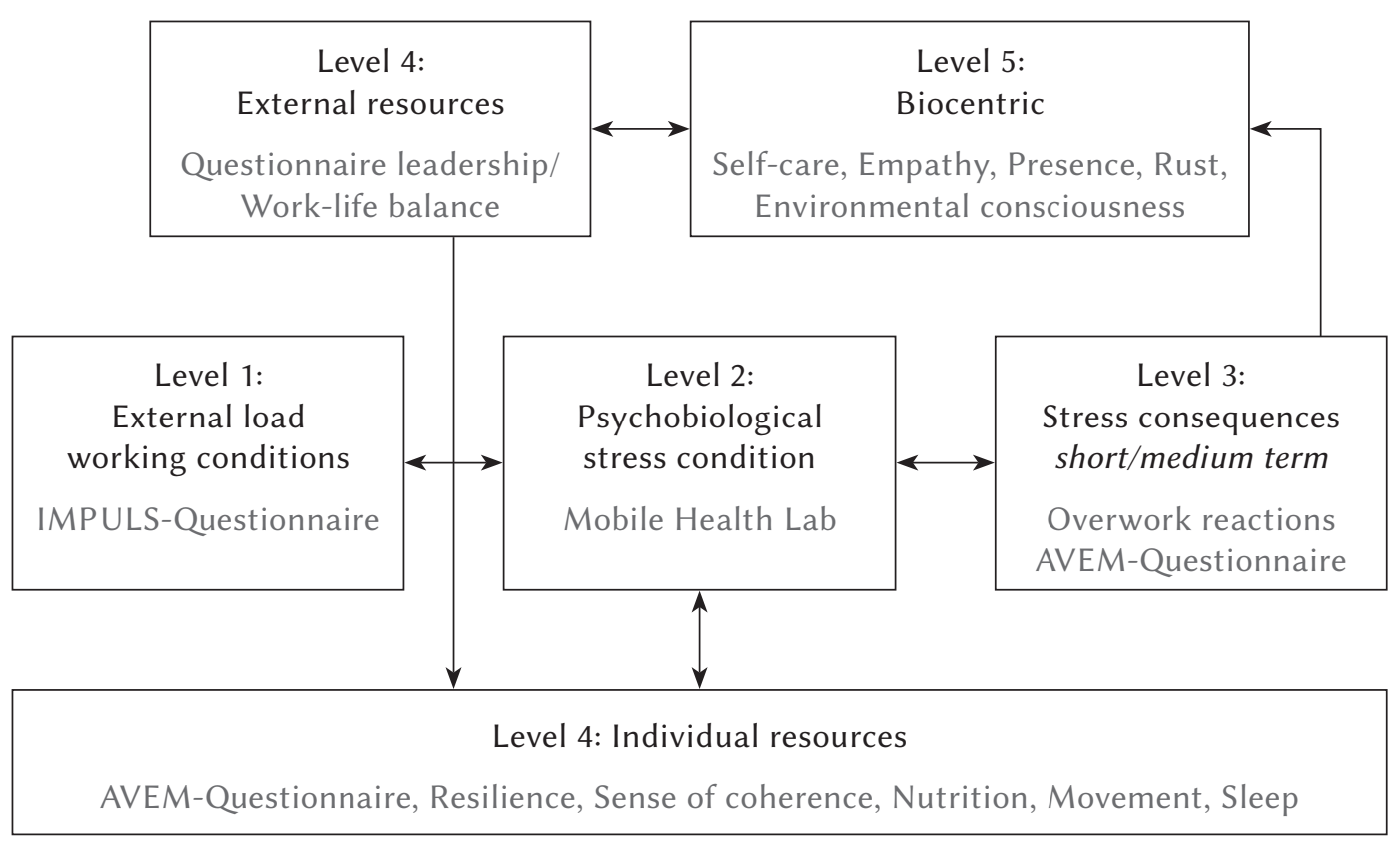

Figure 2. The German bio-centric health management (BHM) model. 
- special stress sequences, e.g. driver behavior,

- stress drivers and constitutional peculiarities (chrono-type), control beliefs (internal/external), dysfunctional stress-related attitudes (Stueck et al., 2019a; Matthews, Desmond, Joyner, Carcary, \& Gilliland, 1997).

\section{Level 4A. Individual resources}

Internal resources are the skills, practices, and abili-

Vahid Delshad,

Hamidreza

Khankeh,

Abbas Ebadi,

Mariola Bidzan, Gholamreza

Ghaedamini Harouni,

Marcus Stueck ties that support own self-regulation:

- resilience and sense of coherence, well-being,

- coping activities (including anger expressive styles, drivers coping),

- dietary attitudes, movement (Matthews et al., 1997).

\section{Level 4B. External resources}

External resources are conditions which support the auto-regulation of workers, e.g. leadership, work-life balance, information health-management, corporate culture, social benefits, working concept (Stueck, 2011; Stueck et al., 2019b).

\section{Table 1}

Psychobiological parameters and devices in second step (psychobiological reactions)

\begin{tabular}{lc}
\hline $\begin{array}{l}\text { Psychobiological } \\
\text { parameter }\end{array}$ & Tool \\
\hline $\begin{array}{l}\text { Hypersensitivity } \\
\text { measurement }\end{array}$ & Hypersensitivity watch \\
Blood pressure & Mercury barometer \\
Pulse curve & Manually \\
Step counting & Fitness watch \\
Heart frequency & HFV device \\
variability & \\
Stress diagnostic test & SDT devices \\
\hline
\end{tabular}

Table 2

Immunological parameters and measurement time will be recorded in the second step as follows

\begin{tabular}{lcc}
\hline $\begin{array}{l}\text { Immunological } \\
\text { parameter }\end{array}$ & Tool & Times \\
\hline & & 4 times \\
Saliva cortisol & Saliva kit & $8,9 \mathrm{am}, 3,6 \mathrm{pm}$ \\
Saliva oxytocin & Saliva kit & $8,9 \mathrm{am}, 3,6 \mathrm{pm}$ \\
Saliva testosterone & Saliva kit & $8,9 \mathrm{am}, 3,6 \mathrm{pm}$ \\
Saliva IgA & Saliva kit & $8,9 \mathrm{am}, 3,6 \mathrm{pm}$ \\
\hline
\end{tabular}

\section{Level 5. Bio-centric acting}

This area has been redesigned for occupational health management and is based on work as an outcome variable in the model.

It is a prerequisite for the development of self-care in its relation to others. In this area, the healthy parts of a person's relationship are diagnosed and promoted in the following three aspects (so-called penguin patterns as the basis of being human):

- relationship with one's own person (including self-respect, self-care, health needs, ability to relax, self-esteem),

- relationship with others, i.e. empathy, trust, affective intelligence, relationship and communication patterns, type of relationship, tolerance, parenting styles,

- relationship with nature: healthy environmental awareness (Maturana \& Varela, 2010; Maturana \& Verden-Zöller, 2008; Stueck \& Villegas, 2017; Toro, 2010; Toro \& Burnod, 2005).

The top 5 levels will be assessed by specific paper and pencil tools that are in English/German and need to be developed and passed psychometrically in the study and the next level specifies psychobiological reactions which play an important role to model psychobiological risk assessment based on theory and studies that have be done (Stück, Rigotti, \& Balzer, 2005) and will be measured with hard electronic devices as explained in the second step below.

\section{SECOND STEP}

\section{Level 2. Psychobiological reactions}

In this step, psychobiological reactions which are an important aspect to model psychobiological risk assessment based on theory and studies that have been done and showed lower physiological arousal and at the same time more changes between arousal and relaxation will be assessed in two phases: 1 - Physiological parameter, 2 - Immunological parameter for the 200 EMS drivers based on BHM pattern, shown in Tables 1 and 2.

Table 1 shows six parameters which will be measured with specific devices during 12-hour shift work.

Table 2 shows the four hormones (cortisol, oxytocin, testosterone and $\operatorname{Ig} \mathrm{A}$ ) that will be recorded four times during 12-hour shift in EMS drivers (Stueck et al., 2020).

Aims of the study are:

- assessing the face and the content validity of the BHM battery,

- assessing the construct validity of the BHM battery,

- assessing the reliability of the BHM battery using the internal consistency and the stability assessment methods, 
- assessing the feasibility of the BHM battery,

- determining the floor and the ceiling effects,

- developing a BHM battery scoring system,

- modeling of bio-centric health management system. Therefore, this paper tries to present how to develop a validated test battery for assessing bio-centric health management based on the psychobiological risk assessment model in an interdisciplinary team and cross-cultural approach, consisting of researchers from Iran and Germany.

\section{PARTICIPANTS AND PROCEDURE}

The study will be conducted in two methodological and modeling phases, in which the authors will be investigating all the aspects of the BHM.

\section{METHODOLOGICAL PHASE}

In the first step, paper and pen tools (questionnaires) will be developed for every independent variable, based on the BHM model.

Then questionnaires will be forward-backward translated based on the WHO research tools guideline (Process of translation and adaptation of instruments) (WHO, 2020).

Then face, content, structural validity and reliability will be assessed, as explained below.

\section{Study population}

The study population consists of all EMS drivers in Tehran who will be selected using purposeful sampling from all EMS stations in Tehran. Sampling will be done with maximum variation respecting male EMS drivers: age, job, educational, and economic status. Inclusion criteria are: age between 18 and 50, experience of 2 years or more, no history of hospitalization for psychiatric disorders, and absence of significant losses 6 months prior to the start of the study.

The focus of this phase is on analyzing the psychometric properties of the battery.

\section{Face validity assessment}

Cognitive interview will be used for face validity assessment. In this step, participants will be asked to comment on the difficulty, appropriateness, and clarity of the items of the paper and pencil tools (Rose, Koshman, Ritchie, \& Sheldon, 2009).

\section{Content validity assessment}

Content validity will also be assessed using both qualitative and quantitative methods. In the qualitative content validity assessment, ten experts will be selected among experts in instrument development, EMS trainers, EMS experts with more than 10 years of working experience, a psychologist, a psychiatrist, psychiatric nurses and epidemiologists; they will be asked to assess the appropriateness of wording, grammar, item allocation, and scaling of the items. Also, quantitative face validity will be assessed using the content validity ratio (CVR) and content validity index (CVI) (Munro, 2005). For CVR calculation, the same experts will be invited to assess the essentiality of the items. Items which are determined by at least nine out of the ten experts will be kept in the questionnaire (Ayre \& Scally, 2014). To calculate the CVI, the same 10 experts will be invited to rate the relevance of each item based on a four-point scale from 1 (irrelevant) to 4 (completely relevant). Then, the number of experts who will rate the relevance of the item as 3 or 4 will be divided by the total number of the experts to calculate CVI. CVI values greater than 0.78 will be considered acceptable (Hyrkäs, Appelqvist-Schmidlechner, \& Oksa, 2003).

\section{Construct validity assessment}

The construct validity of the Health Cube Battery (HCB) will be assessed by using exploratory factor analysis.

\section{Sample size and sampling strategy}

In this study, for any variable a sample of at least 30 is needed and as there are 5 variables ( 4 independent variables and one dependent variable), not less than 200 EMS drivers should be selected for participation (Munro, 2005).

Accordingly, based on the total numbers of EMS stations in Tehran, a proportionate number of EMS drivers will be recruited from each station through purposeful and quota sampling. Participants who fail to answer more than $11 \%$ of HCB items will be excluded (Nulty, 2008).

Confirmatory factor analysis will be done to extract the latent constructs of the HCB. Latent constructs will be derived from the likelihood and varimax rotation (Nia et al., 2014). Factor loading will be set at 0.4 and greater and thus, items with communalities lower than 0.5 will be deleted from the factor analysis (Samitsch, 2014). We will use confirmatory factor analysis to evaluate the most common goodness of fit indices in the next phase of construct validity. The most common goodness of fit indices include root mean score error of approximation (RMSEA), comparative fit index (CFI), adjusted goodness of fit index (AGFI), minimum discrepancy function divided by degrees of freedom (CMIN/DF), and normal fit index (NFI) (Meyers, Gamst, \& Guarino, 2016).
Modeling of biocentric health management in Iran EMS 


\section{Reliability assessment}

The reliability of the paper and pencil tools will be evaluated via the internal consistency, stability, and construct reliability assessment methods. In the internal consistency assessment, Cronbach's $\alpha$ values will be calculated for the test battery and its subscales. Values greater than 0.7 are considered acceptable (Mayers, 2013). Also, for the manual and electronic devices that will be used for the measurements in the second step, calibration and structured approval from the factory will be obtained.

Stability of the battery will also be evaluated via the test-retest technique. For stability assessment, 30 EMS drivers will complete the $\mathrm{HCB}$ twice at a two-week interval, and then the intra-class correlation coefficient (ICC) for the questionnaire and its subscales will be calculated using the two-way mixed effects method. Acceptable stability will be considered if the ICC values are greater than 0.75 (Shoukri, 2010). Moreover, for manual and electronic devices in the second step, the test-retest technique will be performed.

Hair and colleagues' technique will be used to assess construct reliability and standard error measurement. Construct reliability values greater than 0.7 are considered acceptable reliability (Hair, Black, Babin, Anderson, \& Tatham, 2013). The feasibility and simplicity of the $\mathrm{HCB}$ will be evaluated by the average time needed for its completion and the percentage of participants who do not respond to each item (Reneman, Dijkstra, Geertzen, \& Dijkstra, 2010). The average time needed for the $\mathrm{HCB}$ completion will be calculated by measuring the time spent by the first fifty EMS drivers on completing it. The non-response rate of the questionnaire will be calculated from the construct validity assessment data.

\section{Determining the floor and the ceiling effects}

When more than $15 \%$ of the participants achieve the lowest and the highest possible scores, floor and ceiling effects are, respectively, considered present. The presence of these effects indicates that the intended instrument has poor content validity (Terwee et al., 2007). The floor and ceiling effects will be computed using the construct validity assessment data.

\section{Developing Health Cube scoring}

The battery items will be scored on a five-point Likert type scale from 5 to 1 , and negatively worded items will be scored reversely, i.e. from 1 to 5 . The standard 0-100 scoring scale will be applied after the weight of each item has been determined. The raw scores of the questionnaire will be converted to $0-100$ scores using the linear transformation equation depicted (Ibrahim \& Madian, 2011)

\section{SECOND PHASE}

In this phase, AMOS software will separate 150 samples (5 Concept) and the SEM model will be used to determine the relationships between each of the independent variables in the research and bio-centric acting based on psychobiological risk assessment (PBRA) using multiple correlation matrixes (Munro, 2005).

Then, by controlling the independent variables, we will investigate the biosensor function as an intermediate variable in this study (Hair, Gabriel, \& Patel, 2014).

\section{Participant timeline}

Based on time schedule for enrolment and assessments 6 months is estimated, which may be expanded due to some unexpected problems.

Figure 3 shows the expectation of the relationship between variables in the study.

\section{Statistical data analysis}

SPSS and AMOS22 will be used for data analysis. Data will be presented as measures of descriptive statistics including mean, standard deviation, standard error measurement and frequencies. Moreover, statistical analyses will be performed using Pearson correlation analysis, paired- and independent sample $t$-tests, Wilcoxon, Cronbach's $\alpha$ test, intraclass correlation coefficient, confirmatory factor analysis and SEM.

\section{DISCUSSION}

Health risk assessment is an important step in healthrelated activities, and thus requires the use of appropriate instruments. The HCB will create the possibility of collecting data about EMS drivers. In this study, a test battery will be developed based on the definition of bio-centric health management. The psychometric properties of the questionnaire will also be assessed. HCB development can be a critical step in assessing and promoting bio-centric acting among EMS drivers. Moreover, BHM modeling can help determine the relationship between the variables and the effect on bio-centric acting of EMS drivers, which is essential for determining how the target group should work to increase their bio-centric acting in BHM. Other scholars can also cross-culturally adapt and use the HCB and our model according to their immediate contexts and culture for other professions.

Based on Bahadori et al. (2016), the number of studies on pre-hospital emergency services conducted in Iran is limited. To promote public health, the consideration of prevention areas, a process of providing pre-hospital emergency services, policy- 


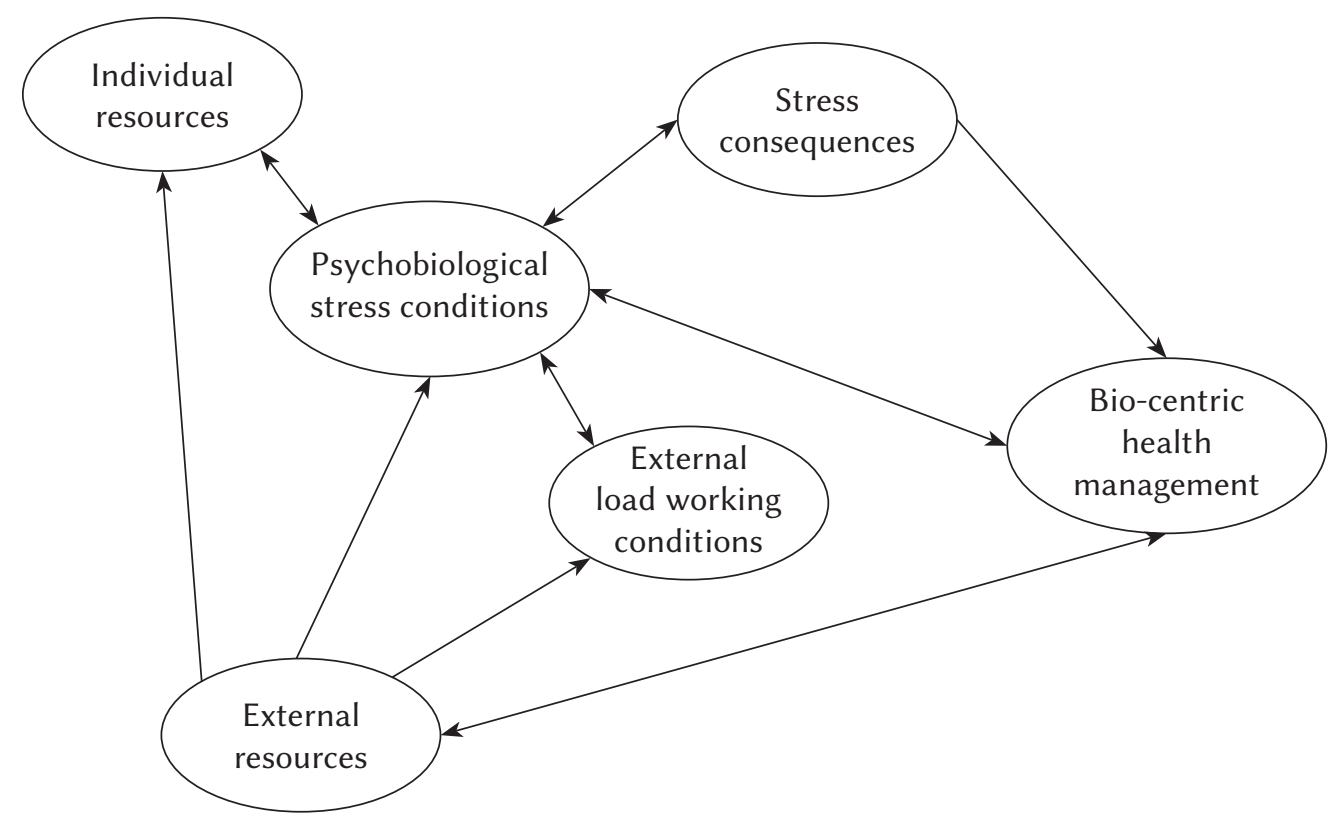

Modeling of biocentric health management in Iran EMS

Figure 3. Expected model of BHM for EMS drivers.

making, foresight, adoption of a systemic view, development of comprehensive research programs and roadmaps, and research needs assessments in prehospital emergency seem to be necessary (Bahadori et al., 2016).

On the one hand, the strengths of this study include the following: this is the first time the BHM has been modeled for EMS drivers, who are workers in high stress environments; the relatively large sample size in the present study; and the biological assessment (physiological and immunological test) performed in the study. On the other hand, the reluctance of some EMS drivers to collaborate with the study and the difficult physic-immunological tests may be the most important study limitations.

Some studies show that more assessment and education are needed for first responders, to prevent road traffic injuries in Iran (Farzinnia, Delshad, Farzinnia, \& Khankeh, 2018). In our study the first important step will be done as a PBRA and in future study based on this model we can evaluate different interventions for EMS drivers and other related professions. The findings will be useful for policy makers and planners who aim to reduce the unsafe behaviors which are dangerous both to the EMS drivers and patients. They can be implemented and integrated in the driving regulations of Iranian EMS drivers, and also in other professions. Additionally, the results will be helpful in exploring the process of PBRA composed of the current psychobiological pattern they feel, the process which they experience, and coping with the symptoms and consequences in this context (Birks \& Mills, 2015).

\section{ETHICS APPROVAL AND CONSENT TO PARTICIPATE}

This $\mathrm{PhD}$ project was evaluated positively by the Ethical Committee and approved by the Health in Emergency and Disaster research center of the University of Social Welfare and Rehabilitation (Approval ID: IR.USWR.REC.1398.145, Approval Date: 2019.11.25), Iran.

This $\mathrm{PhD}$ project was approved by the Ethical Committee in the University of Social Welfare and Rehabilitation (USWR) (Approval ID: IR.USWR. REC.1398.145 Approval Date: 2019.11.25), Iran.

Consent to participate is in written form and was evaluated and approved by the ethical committee as a standard form in USWR university (this project will be done in Iran and the consent letter is in Farsi).

This study is ongoing and has not completed participant recruitment at the time of submission.

\section{References}

Ayre, C., \& Scally, A. J. (2014). Critical values for Lawshe's content validity ratio: Revisiting the original methods of calculation. Measurement and Evaluation in Counseling and Development, 47, 79-86. https://doi.org/10.1177/0748175613513808

Bahadori, M., Ghardashi, F., Izadi, A. R., Ravangard, R., Mirhashemi, S., \& Hosseini, S. M. (2016). Pre-hospital emergency in Iran: a systematic review. Trauma Monthly, 21, e31382. https://doi.org/10.5812/traumamon.31382 
Birks, M., \& Mills, J. (2015). Grounded theory: a practical guide. Los Angeles, CA: Sage.

Compas, B. E. (2006). Psychobiological processes of stress and coping: Implications for resilience in children and adolescents - comments on the papers of Romeo \& McEwen and Fisher et al. Annals of the New York Academy of Sciences, 1094, 226-234. https://doi.org/10.1196/annals.1376.024

Dadashzadeh, A., \& Rahmani, A. (2017). Exposure to stressors among emergency medical technicians
Vahid Delshad,

Hamidreza

Khankeh,

Abbas Ebadi,

Mariola Bidzan, Gholamreza Harouni,

Marcus Stueck
Ghaedamini in pre-hospital emergency departments of East Azerbaijan Province, Iran. Iranian Journal of Emergency Care, 1, 18-27.

Deb, S., Chakraborty, T., Chatterjee, P., \& Srivastava, N. (2008). Job-related stress, causal factors and coping strategies of traffic constables. Journal of the Indian Academy of Applied Psychology, 34, 19-28.

Delshad, V., Sabzalizadeh, S., Moradian, M. J., Safarpour, H., Malekyan, L., Shemshadi, H., \& Beiranvand, R. (2018). Epidemiology of accidents in Tehran emergency medical service during 2012 to 2013. Trauma Monthly, 23, e61871. https://doi. org/10.5812/traumamon.61871

Delshad, V., Shemshadi, H., Moradian, M. J., Ahmadi, S., Malkyan, L., \& Sabzalizadeh, S. (2016). The effect of applying global positioning system in ambulances on response time of Tehran emergency medical service. Health in Emergencies and Disasters Quarterly, 1, 125-128. https://doi.org/10.15412/J. HDQ.09010302

Ebrahimian, A., Seyedin, H., Jamshidi-Orak, R., \& Masoumi, G. (2014). Exploring factors affecting emergency medical services staffs' decision about transporting medical patients to medical facilities. Emergency Medicine International, 2014, article 215329. https://doi.org/10.1155/2014/215329

Entekhab (2020). Head of Tehran EMS report. Retrieved from https://www.entekhab.ir/fa/news/. https://www.entekhab.ir/fa/news/

Farzinnia, B., Delshad, V., Farzinnia, M., \& Khankeh, H. (2018). Facilitators, causes and lesson learnt from two bus incident in Tehran-Qom Highway: a case study in Iran. Trauma Monthly, 23, e63249. https:// doi.org/10.5812/traumamon.63249

Fox, G. K., Bashford, G. M., \& Caust, S. L. (1992). Identifying safe versus unsafe drivers following brain impairment: The Coorabel Programme. Disability and Rehabilitation, 14, 140-145. https://doi. org/10.3109/09638289209165850

Golmohamadi, R., Damyar, N., Mohamadfam, I., \& Faradmal, J. (2013). Study of occupational stress among Hamadan city-bus drivers, 2011. Tolooebehdasht, 12, 24-32.

Hair, J. F., Black, W. C., Babin, B. J., Anderson, R. E., \& Tatham, R. L. (2013). Multivariate data analysis. Essex: Pearson Education Limited.

Hair, J. F., Gabriel, M., \& Patel, V. (2014). AMOS covariance-based structural equation modeling
(CB-SEM): Guidelines on its application as a marketing research tool. Brazilian Journal of Marketing, 13, 44-55. https://doi.org/10.5585/remark. v13i2.2718

Hajiamini, Z., Cheraghalipour, Z., Azad Marzabadi, E., Ebadi, A., \& Norouzi Koushali, A. (2011). Comparison of job stress in military and non-military drivers in Tehran. Iranian Journal of Military Medicine, 13, 25-30.

Hennessy, D. A., Jakubowski, R. D., \& Leo, B. (2016). The impact of primacy/recency effects and hazard monitoring on attributions of other drivers. Transportation Research Part F: Traffic Psychology and Behaviour, 39, 43-53. https://doi.org/10.1016/j. trf.2016.03.001

Heydari, A., Rad, M., Ghasemi, M. R., Sabzevari, M. T., \& Rad, M. (2014). Challenges ahead of emergency medical technician graduates in the workplace in Iran: a qualitative study. Journal of Allied Health, 43, E19-E24.

Hyrkäs, K., Appelqvist-Schmidlechner, K., \& Oksa, L. (2003). Validating an instrument for clinical supervision using an expert panel. International Journal of Nursing Studies, 40, 619-625. https://doi. org/10.1016/s0020-7489(03)00036-1

Ibrahim, E. M., \& Madian, A. (2011). Impact of hepatitis $\mathrm{C}$ on health-related quality of life in Egypt. Journal of American Sciences, 7, 430-439.

Koslowsky, M., Kluger, A. N., \& Reich, M. (2013). Commuting stress: Causes, effects, and methods of coping. Berlin: Springer Science \& Business Media.

Matthews, G., Desmond, P. A., Joyner, L., Carcary, B., \& Gilliland, K. (1997). A comprehensive questionnaire measure of driver stress and affect. In T. Rothengatter \& E. Carbonell Vaya (Eds.), Traffic and transport psychology: Theory and application (pp. 317-324). Amsterdam: Pergamon.

Maturana, H. R., \& Varela, F. J. (2010). Der Baum der Erkenntnis. Die biologischen Wurzeln menschlichen Erkennens (3. Auflage) [The tree of knowledge. The biological roots of human knowledge (3rd edition]. Frankfurt am Main: S. Fischer Verlag.

Maturana, H. R., \& Verden-Zöller, G. (2008). The origin of humanness in the biology of love. Exeter: Imprint Academic.

Mayers, A. (2013). Introduction to statistics and SPSS in psychology. London: Pearson Higher Ed.

Meyers, L. S., Gamst, G., \& Guarino, A. J. (2016). Applied multivariate research: Design and interpretation. Thousand Oaks, CA: Sage Publications.

Moradi, F., Jafari, H. A., \& Hendiani, A. (2016). Investigating the effective factors on task force occupational stress of firefighting organization of Tehran municipality. International Journal of Humanities and Cultural Studies, 2, 622-631.

Munro, B. H. (2005). Statistical methods for health care research (Vol. 1). Philadelphia, PA: Lippincott Williams \& Wilkins. 
National EMS (2020). Number of missions in last 24 hours based on national website report. Retrieved from http://www.115.ir

Nia, H. S., Ebadi, A., Lehto, R. H., Mousavi, B., Peyrovi, H., \& Chan, Y. H. (2014). Reliability and validity of the Persian version of Templer Death Anxiety Scale-Extended in veterans of Iran-Iraq warfare. Iranian Journal of Psychiatry and Behavioral Sciences, 8, 29-37.

Nulty, D. D. (2008). The adequacy of response rates to online and paper surveys: What can be done? Assessment \& Evaluation in Higher Education, 33, 301314. https://doi.org/10.1080/02602930701293231

Reneman, M. F., Dijkstra, A., Geertzen, J. H. B., \& Dijkstra, P. U. (2010). Psychometric properties of chronic pain acceptance questionnaires: a systematic review. European Journal of Pain, 14, 457465. https://doi.org/10.1016/j.ejpain.2009.08.003

Reschke, K., Kranich, U., Stoeber, F., \& Neubauer, S. (2019). Training of resiliency for high stressed groups: The development and evaluation of stress management programs for elderly people and for drivers. Proceedings of the IV Leipzig-Evora Scientific Meeting in Psychology, 1st International Scientific Meeting in Psychology, 17-25.

Rose, M. S., Koshman, M. L., Ritchie, D., \& Sheldon, R. (2009). The development and preliminary validation of a scale measuring the impact of syncope on quality of life. Europace, 11, 1369-1374. https:// doi.org/10.1093/europace/eup106

Rutkow, L., Gable, L., \& Links, J. M. (2011). Protecting the mental health of first responders: Legal and ethical considerations. The Journal of Law, Medicine \& Ethics, 39, 56-59. https://doi.org/10.1111/j.1748720X.2011.00567.x

Saberinia, E., Jannat, F., \& Aminizadeh, M. A. A. (2019). The effect of mindfulness-based cognitive therapy on anxiety of pre-hospital emergency staff. Archives of Rehabilitation, 20, 340-349. https://doi. org/10.32598/rj.20.4.340

Samitsch, C. (2014). Data quality and its impacts on decision-making: How managers can benefit from good data. Berlin: Springer.

Schaarschmidt, U. (2006). AVEM - ein persönlichkeitsdiagnostisches Instrument für die berufsbezogene Rehabilitation [AVEM - a personality diagnostic instrument for professional related rehabilitation]. In Arbeitskreis Klinische Psychologie in Der Rehabilitation BDP (Ed.), Psychologische Diagnostik - Weichenstellung Für Den Reha-Verlauf [Psychological diagnostics - setting the course for the Rehab process] (pp. 59-82). Bonn: Deutscher Psychologen Verlag.

Shoukri, M. M. (2010). Measures of interobserver agreement and reliability. Cleveland, $\mathrm{OH}$ : CRC Press.

Sterud, T., Ekeberg, Ø., \& Hem, E. (2006). Health status in the ambulance services: a systematic review. BMC Health Services Research, 6, 82. https:// doi.org/10.1186/1472-6963-6-82
Stück, M., Rigotti, T., \& Balzer, H. U. (2005). Wie reagieren Lehrer bei Belastungen? Berufliche Bewältigungsmuster und psychophysiologische Korrelate [How do teachers react to stress? Work related coping styles and psychophysiological correlates]. Psychologie in Erziehung und Unterricht, 52, 250-260. Stueck, M. (2011). The concept of system-related stress reduction (SYSRED) in educational fields. Problems of Education in the 21st Century, 29, 119-134.

Stueck, M., Delshad, V., Roudini, J., Khankeh, H., Ranjbar, M., Reschke, K., Villegas, A., Balzer, H. U., Matthews, G., \& Sack, U. (2019a). Health Cube with school of empathy and stress reduction for Iranian drivers: New tools for traffic psychology and biocentric health management in Iran. Modern Care Journal, 16, e90632. https://doi.org/10.5812/modernc. 90632

Stueck, M., \& Villegas, A. (2017). Evidence-based BIODANZA programmes for children (TANZPRO-Biodanza) in schools and kindergardens: Some effects on psychology, physiology, hormones, and the immune system. In V. Karkou, S. Oliver, \& S. Lycouris (Eds.), The Oxford handbook of dance and wellbeing (pp. 77-98). New York: Oxford University Press.

Stueck, M., Witruk, E., \& Utami, S. (2019b). Der Gesundheitswürfel: Ein Instrument zur Psychischen Gefährdungs- und Ressourcenbeurteilung im Rahmen des Biozentrischen und Betrieblichen Gesundheitsmanagements in Unternehmen und Institutionen [The Health Cube: an instrument for psychological risk and resource assessment within the framework of biocentric and occupational health management in companies and institutions]. In E. Witruk \& S. Utami (Eds.), Educational and Rehabilitation Psychology, Vol. 8: Traumatic Experiences and Dyslexia (pp. 1-18). Berlin: Peter Lang.

Stueck, M., Witruk, E., \& Utami, D. S. (2020). The biocentric approach in the occupational psychology. Unpublished manuscript.

Terwee, C. B., Bot, S. D. M., de Boer, M. R., van der Windt, D. A., Knol, D. L., Dekker, J., Bouter, L. M., \& de Vet, H. C. W. (2007). Quality criteria were proposed for measurement properties of health status questionnaires. Journal of Clinical Epidemiology, 60, 34-42. https://doi.org/10.1016/j.jclinepi.2006.03.012

Toro, R. (2010). Das System Biodanza [The Biodanza system]. Hannover: Tinto-Verlag.

Toro, R., \& Burnod, Y. (2005). A morphogenetic model for the development of cortical convolutions. Cerebral Cortex, 15, 1900-1913. https://doi. org/10.1093/cercor/bhi068

WHO (2020). Tools guideline (Process of translation and adaptation of instruments). Retrieved from https://www.who.int/substance_abuse/research_ tools/translation/en/
Modeling of biocentric health management in Iran EMS 